\title{
The Abundance of Nonphosphorylated Tau in Mouse and Human Tauopathy Brains Revealed by the Use of Phos-Tag Method
}

Taeko Kimura, ${ }^{*}$ Hiroyuki Hatsuta, ${ }^{\dagger}$ Masami Masuda-Suzukake, ${ }^{\ddagger}$ Masato Hosokawa, ${ }^{\ddagger}$ Koichi Ishiguro, ${ }^{\S}$ Haruhiko Akiyama, Shigeo Murayama, ${ }^{\dagger}$ Masato Hasegawa, ${ }^{\ddagger}$ and Shin-ichi Hisanaga*

From the Department of Biological Sciences, * Laboratory of Molecular Neuroscience, Tokyo Metropolitan University, Hachioji, Tokyo; the Department of Neuropathology, ${ }^{\dagger}$ Tokyo Metropolitan Institute of Gerontology, Itabashi, Tokyo; the Department of Dementia and Higher Brain Function, ${ }^{\ddagger}$ Tokyo Metropolitan Institute of Medical Science, Setagaya, Tokyo; and the Department of Neurology, ${ }^{\S}$ Graduate School of Medicine, Juntendo University, Tokyo, Japan

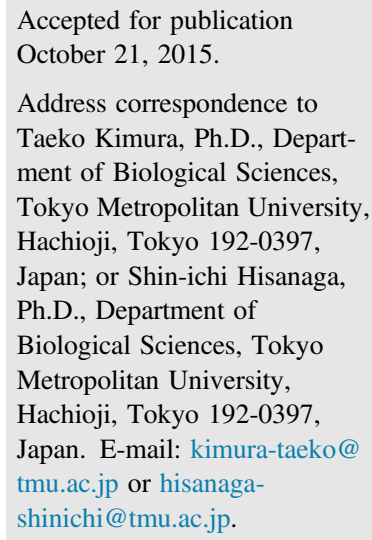

\begin{abstract}
Tauopathies are neurodegenerative diseases characterized by aggregates of hyperphosphorylated tau. Previous studies have identified many disease-related phosphorylation sites on tau. However, it is not understood how tau is hyperphosphorylated and what extent these sites are phosphorylated in both diseased and normal brains. Most previous studies have used phospho-specific antibodies to analyze tau phosphorylation. These results are useful but do not provide information about nonphosphorylated tau. Here, we applied the method of Phos-tag SDS-PAGE, in which phosphorylated tau was separated from nonphosphorylated tau in vivo. Among heterogeneously phosphorylated tau species in adult mouse brains, the nonphosphorylated 0N4R isoform was detected most abundantly. In contrast, perinatal tau and tau in cold water-stressed mice were all phosphorylated with a similar extent of phosphorylation. In normal elderly human brains, nonphosphorylated ON3R and ON4R tau were most abundant. A slightly higher phosphorylation of tau, which may represent the early step of hyperphosphorylation, was increased in Alzheimer disease patients at Braak stage V. Tau with this phosphorylation state was pelleted by centrifugation, and sarkosyl-soluble tau in either Alzheimer disease or corticobasal degeneration brains showed phosphorylation profiles similar to tau in normal human brain, suggesting that hyperphosphorylation occurs in aggregated tau. These results indicate that tau molecules are present in multiple phosphorylation states in vivo, and nonphosphorylated forms are highly expressed among them. (Am J Pathol 2016, 186: 398-409; http://dx.doi.org/10.1016/j.ajpath.2015.10.009)
\end{abstract}

Alzheimer disease (AD) is the most common form of dementia in the elderly and is characterized by senile plaques composed of amyloid $\beta$ peptides and neurofibrillary tangles (NFTs) that consist of hyperphosphorylated forms of tau protein. ${ }^{1,2}$ NFTs are also found in many other neurodegenerative diseases, including corticobasal degeneration (CBD), progressive supranuclear palsy, Pick disease, and frontotemporal dementia and parkinsonism linked to chromosome 17 (FTDP-17). These diseases are collectively referred to as tauopathies. ${ }^{3}$ Because FTDP-17 is an inherited disease with mutations in the tau gene $M A P T,{ }^{4-6}$ mutation of tau is sufficient to cause neurodegeneration and dementia. Most mutations are found in the microtubule (MT)-binding region of tau, but it is not known why mutations induce diseases. Tau in NFTs is hyperphosphorylated. Previous studies showed that hyperphosphorylated tau has a reduced MT-binding ability and a propensity to self-aggregate into paired helical filaments, a component of NFTs, ${ }^{7,8}$ but how tau becomes hyperphosphorylated during $\mathrm{AD}$ progression is not known. More than 40 phosphorylation sites are found in NFT-associated tau, with phosphorylation at Ser/Thr-Pro

Supported by Ministry of Education, Culture, Sports, Science and Technology in Japan grants-in-aid for scientific research on priority area 25290024, 26117004 (S.H.), and 15K18366 (T.K.)

Disclosures: None declared. 
sequences being characteristic. ${ }^{9,10}$ It is still unclear whether there is disease type-specific phosphorylation of tau. It is also important to analyze tau phosphorylation more quantitatively and combinatorially to further understand the pathologic mechanism(s) of tauopathies.

Tau is a MT-associated protein, whose function is principally to polymerize and stabilize MTs in axons. ${ }^{11}$ Tau consists of the $\mathrm{N}$-terminal projection and C-terminal MT-binding domains. There are isoforms of tau with three or four MT-binding repeats, $3 \mathrm{R}$ or $4 \mathrm{R}$, respectively, in the MT-binding domain, which are generated by alternative splicing of exon $10 .^{12}$ In addition, another alternative splicing in the $\mathrm{N}$-terminal two insertions (exons 2 and 3) produces three isoforms, resulting in a total of six isoforms of tau. Expression of isoforms is regulated developmentally; $3 \mathrm{R}$ tau is expressed in fetal to neonatal mouse brain, and 4R tau is expressed in adult mouse brain. ${ }^{13,14}$ In adult human brain, all six isoforms are expressed. ${ }^{15}$ Although $4 \mathrm{R}$ tau has a stronger MT-binding ability than 3R tau, it is not known whether these isoforms have different functions in vivo. ${ }^{16}$ Tau is also phosphorylated physiologically. ${ }^{17}$ This phosphorylation regulates the MT-assembling activity of tau in a site-specific manner; phosphorylation in the MT-binding repeats abolishes the MT-binding activity, whereas that in the flanking regions only partially reduces this activity. ${ }^{16,18}$ Many kinases, including proline-directed protein kinases such as glycogen syntase kinase $3 \beta$ or cyclin-dependent kinase 5, phosphorylate tau at multiple sites both commonly and distinctly, ${ }^{10}$ but the precise role of sitespecific phosphorylation is not yet understood.

Both normal and abnormal phosphorylation of tau were studied with the use of phospho-specific antibodies. Currently, many phospho-specific tau antibodies are commercially available. Although these antibodies are powerful and sensitive enough to detect a small change in phosphorylation at a particular site, ${ }^{19}$ it is difficult to determine the absolute ratio of phosphorylation and to make quantitative comparisons between experiments. In addition, many site-specific antibodies are generated to produce a complete phosphorylation profile. This is time consuming and expensive, and there are sometimes problems with specificity because of closely located phosphorylation sites. Thus, another simple and quantitative method for analysis of phosphorylated tau is needed. ${ }^{20}$

Phos-tag SDS-PAGE is an established method that shifts the mobility of all phosphorylated proteins, depending on their number and sites of phosphorylation. ${ }^{21}$ We have previously used this method for quantitative measurement of combinatory phosphorylation of the p35 cyclin-dependent kinase 5 activator in vivo. ${ }^{22}$ In this study, we characterized phospho-tau species in brains of wild-type and AD model mice and in brains of human $\mathrm{AD}$ and $\mathrm{CBD}$ patients with the use of Phos-tag SDS-PAGE. The analyses revealed a number of interesting insights about in vivo phosphorylation of tau, indicating the usefulness of this method for studying tau phosphorylation.

\section{Materials and Methods}

\section{Antibodies and Chemicals}

Anti-Tau Ab-2 (Tau5), anti-phospho-Tau AT8, and antihuman tau HT7 were purchased from Thermo Fisher Scientific (Fremont, CA). Anti-unphospho-Tau (Tau-1), RD3, and RD4 were obtained from Millipore (Darmstadt, Germany). Phospho-Ser404 (P-S404) was obtained from Abcam (Cambridge, UK). Tau-C, the antibody against the C-terminal sequence of tau (amino acids 422 to 438), was described previously. ${ }^{23}$ Anti-4R was described previously. ${ }^{24}$ 4-(2-aminoethyl)-benzenesulfonyl fluoride hydrochloride (AEBSF), Phos-tag acrylamide, microcystin LR, and alkaline phosphatase were purchased from Wako Chemicals (Osaka, Japan). Leupeptin was obtained from the Peptide Institute (Osaka, Japan).

\section{Expression and Purification of Tau}

Recombinant human tau, 0N3R, 0N4R, 1N3R, 1N4R, 2N3R, and 2N4R, and mouse tau, 0N3R, 0N4R, and 1N4R, were synthesized in Escherichia coli cultures and were purified as described previously. ${ }^{25}$ Protein concentration was determined by Coomassie brilliant blue staining of the polyacrylamide gels with the use of bovine serum albumin as a standard.

\section{Preparation of Extracts from Mouse and Human Brains}

All animal studies were conducted in compliance with the guidelines for animal experimentation of Tokyo Metropolitan University and the Tokyo Metropolitan Institute of Medical Science. The P301L tau transgenic mouse line $\left(\right.$ JNPL3) ${ }^{26}$ was obtained from Taconic (Hudson, NY). ${ }^{27}$ The $5 \times$ FAD mice $^{28}$ that have transgenes of human amyloid precursor protein and presenilin-1 with five mutations found in familial $\mathrm{AD}$ were obtained from The Jackson Laboratory (Bar Harbor, ME). The mice were housed in cages of two to five littermates with access to food and water ad libitum in an environment subjected to a 12-hour light/dark cycle in the animal facility of the Tokyo Metropolitan Institute of Medical Science. C57BL/6J mice were obtained from Sankyo Labo Service (Tokyo, Japan). C57BL/6J mice at 96 weeks of age (96W) were provided by the Tokyo Metropolitan Institute of Gerontology. The cerebral cortices from mouse brains were individually homogenized in $10 \mathrm{mmol} / \mathrm{L}$ Tris- $\mathrm{HCl}$ ( $\mathrm{pH} 7.4), 1$ $\mathrm{mmol} / \mathrm{L}$ EGTA, $0.8 \mathrm{~mol} / \mathrm{L} \mathrm{NaCl}, 10 \%$ sucrose, $10 \mathrm{mmol} / \mathrm{L}$ $\mathrm{NaF}, 10 \mathrm{mmol} / \mathrm{L} \beta$-glycerophosphate, $0.4 \mathrm{mmol} / \mathrm{L}$ AEBSF, $10 \mu \mathrm{g} / \mathrm{mL}$ leupeptin, and $1 \mathrm{mmol} / \mathrm{L}$ dithiothreitol. After centrifugation at $15,000 \times g$ for 20 minutes, the supernatant fluid was used as the brain extract. Sarkosyl-insoluble and -soluble fractions were separated as described. ${ }^{29}$

Human brain tissues were obtained from the brain bank of the Tokyo Metropolitan Institute of Gerontology, and the study that used human brain tissues was approved by the 
Table 1 Summary of Cases

\begin{tabular}{lllll}
\hline & & & & NFT, Braak \\
Case & Age, years & Sex & PMI, hours & stage \\
\hline a & 77 & F & 17.1 & I \\
b & 76 & F & 20.5 & I \\
c & 75 & M & 31.5 & II \\
d & 75 & F & 32.3 & I \\
e & 77 & F & 9.0 & I \\
f & 76 & M & 3.8 & II \\
g & 79 & F & 3.4 & V \\
h & 80 & F & 6.7 & V \\
i & 77 & M & 11.4 & V \\
j & 76 & M & 15.8 & VI \\
k & 76 & M & 15.8 & VI \\
l & 77 & F & 6.7 & VI \\
m & 79 & M & 12.3 & II \\
n & 74 & F & 53.5 & I \\
o & 69 & F & 3.3 & I \\
\hline
\end{tabular}

F, female; M, male; NFT, neurofibrillary tangle; PMI, postmortem interval.

ethics committees of Tokyo Metropolitan University and the Tokyo Metropolitan Institute of Gerontology. Detailed descriptions of the subjects are given in Table 1. Braak stages were determined as described. ${ }^{30,31}$ The brain extracts were prepared from blocks of frozen human brains, consisting of the temporal cortical and precentral gyrus regions, by homogenizing in $10 \mathrm{mmol} / \mathrm{L}$ Tris- $\mathrm{HCl}(\mathrm{pH} 7.4), 10$ $\mathrm{mmol} / \mathrm{L} \mathrm{NaF}, 10 \mathrm{mmol} / \mathrm{L} \beta$-glycerophosphate, $0.4 \mathrm{mmol} / \mathrm{L}$ AEBSF, $10 \mu \mathrm{g} / \mathrm{mL}$ leupeptin, and $1 \mathrm{mmol} / \mathrm{L}$ dithiothreitol. Sarkosyl-insoluble and -soluble fractions were prepared as described. $^{29}$

\section{Treatment of Mice with Ice-Cold Water}

Mice were immersed up to the neck in ice-cold water for 5 minutes. Then, they were gently wiped dry and returned to their cages at room temperature. ${ }^{32,33}$ After 20 and 90 minutes, the mice were sacrificed by cervical dislocation, and the brains were dissected.

\section{Laemmli SDS-PAGE, Phos-Tag SDS-PAGE, Immunoblotting, and Densitometric Scans}

Laemmli SDS-PAGE was performed with the use of $12.5 \%$ (w/v) polyacrylamide gels, and Phos-tag SDS-PAGE was performed with the use of $7.5 \%(\mathrm{w} / \mathrm{v})$ polyacrylamide gels that contained $50 \mu \mathrm{mol} / \mathrm{L}$ Phos-tag acrylamide and 150 $\mu \mathrm{mol} / \mathrm{L} \mathrm{MnCl}_{2}$ as described. ${ }^{22,25,34}$ For immunoblotting, proteins separated in a gel were transferred to a polyvinylidene difluoride membrane with the use of a submerged blotting apparatus, and immunoreaction was visualized by exposure on X-ray film (Fuji Film, Tokyo, Japan) with the use of the Enhanced Chemiluminescence Detection kit (GE Healthcare, Tokyo, Japan) as described previously. ${ }^{25}$ The band intensity and banding profiles were analyzed with ImageJ version 64 software (NIH, Bethesda,
MD; http://imagej.nih.gov/ij) after densitometric scanning of X-ray films.

\section{Results \\ Phosphorylation States of Tau in Mouse Brain Analyzed by Phos-Tag SDS-PAGE}

We analyzed phosphorylation of tau in mouse brains by immunoblotting with a phosphorylation-independent antibody against Tau (Tau5) after Phos-tag SDS-PAGE. Tau in adult mice at $10 \mathrm{~W}$ separated into more than four bands with a smear background in Phos-tag SDS-PAGE (Figure 1A). The electrophoretic mobility was compared with recombinant mouse tau $0 \mathrm{~N} 3 \mathrm{R}, 0 \mathrm{~N} 4 \mathrm{R}$, and $1 \mathrm{~N} 4 \mathrm{R}$. The lowest strong band migrated slightly faster than recombinant $0 \mathrm{~N} 4 \mathrm{R}$ tau. To see which tau bands were phosphorylated, we treated the brain extract with alkaline phosphatase and ran it in parallel with an untreated sample on Phos-Tag SDS-PAGE (Figure 1B). The mobility of the lowest band was not changed by this treatment, but the upper bands shifted down to the second band from the bottom, which migrated slightly faster than recombinant $1 N 4 R$ tau. Given that the $4 R$ tau isoforms $0 \mathrm{~N}$ and $1 \mathrm{~N}$ are main isoforms expressed in adult mouse, ${ }^{13,14}$ these two fastest migrating tau bands in the alkaline phosphatase-treated sample likely correspond to nonphosphorylated 0N4R and 1N4R tau. However, most of the upper bands in the untreated extract were phosphorylated forms of tau appeared at the second band from the bottom. Their densitometric scan profiles are also shown (Figure 1). The lowest band accounted for approximately $15.5 \% \pm 1.5 \%(n=3)$ of total tau in the brain extract before the alkaline phosphatase treatment. These results indicate that tau isoforms are differentially phosphorylated; the ON4R isoform is primarily nonphosphorylated, and $1 \mathrm{~N} 4 \mathrm{R}$ tau is more phosphorylated in mouse brain. The phosphorylation of tau changes with brain development. ${ }^{35-38}$ We examined the phosphorylation status of tau in mouse brains at postnatal day 2 (P2) and 96W in addition to the $10 \mathrm{~W}$ brains with the use of Phos-tag SDS-PAGE. Tau at P2 showed considerably slower migration than tau at $10 \mathrm{~W}$ in Phos-tag SDS-PAGE immunoblotting (Figure 1C), confirming higher phosphorylation of tau in early postnatal days. In contrast, the banding pattern of tau in older mice at $96 \mathrm{~W}$ was similar to that at 10W (Figure 1C). The main isoforms of tau expressed in brain change from $3 \mathrm{R}$ to $4 \mathrm{R}$ at around $\mathrm{P} 20 .^{14,39}$ To confirm that the difference in the phosphorylation state between $\mathrm{P} 2$ and $10 \mathrm{~W}$ tau is not because of a difference in isoforms (ie, $3 \mathrm{R}$ versus $4 \mathrm{R}$ ), we performed immunoblots with 3R- and 4R-specific antibodies, RD3 and RD4, respectively (Figure 1C). ${ }^{40,41}$ The results were consistent with previous reports that the $3 \mathrm{R}$ isoform is expressed during perinatal days and that $4 \mathrm{R}$ is expressed in adult mouse brains. ${ }^{13,14}$ Longer exposure of the blot for RD3 revealed the existence of a small amount of $3 \mathrm{R}$ tau in brain at $10 \mathrm{~W}$ with migration similar to $4 \mathrm{R}$ tau (Figure 1C), indicating that, like $4 \mathrm{R}$ tau, $3 \mathrm{R}$ tau is less phosphorylated in adult brain. 
A

B

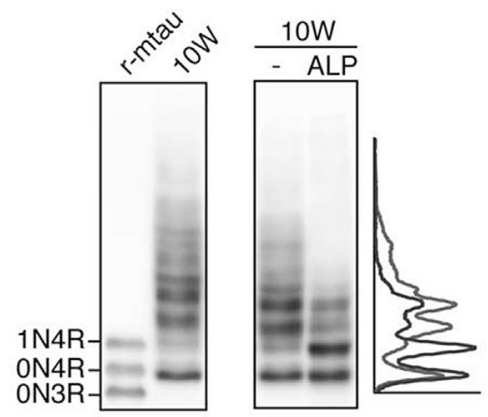

C

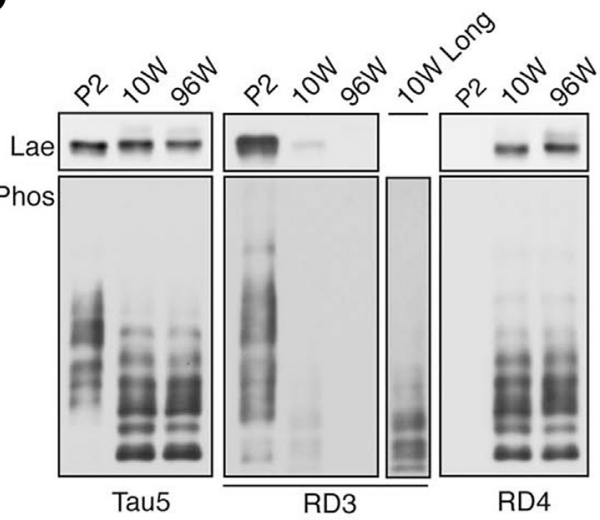

D

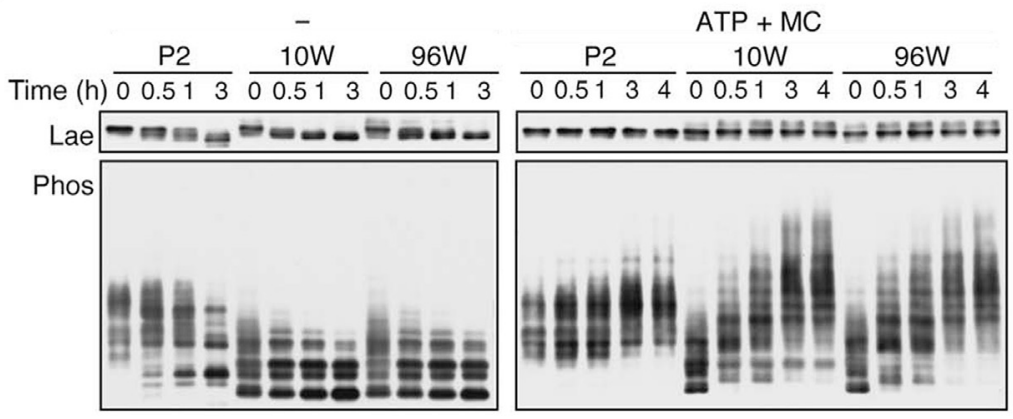

E

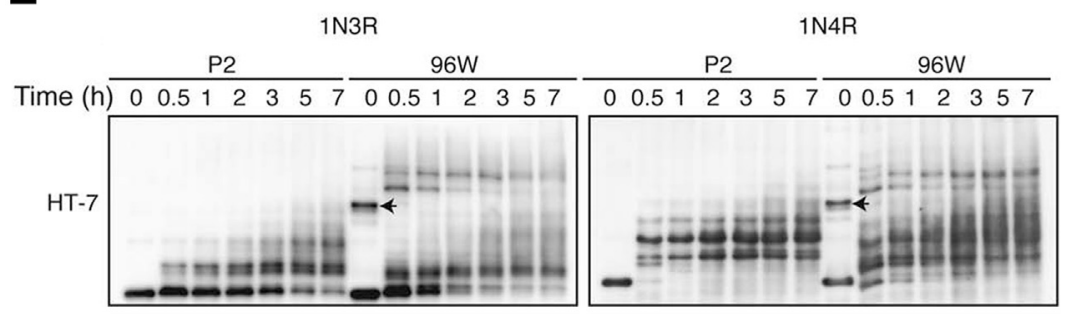

Figure 1 Phosphorylation states of tau in mouse brain analyzed by Phos-tag SDS-PAGE. A: An immunoblot of tau in adult mouse brain with the use of Phos-tag SDS-PAGE. A brain extract was prepared from a mouse at $10 \mathrm{~W}$ and was immunoblotted with Tau 5 after Phos-tag SDS-PAGE. Mouse recombinant tau isoforms ON3R, ON4R, and 1N4R are shown in the left lane ( $r$-mtau). B: Dephosphorylation of mouse tau with ALP. The mouse brain extract was incubated with $0.6 \mathrm{U} / \mu \mathrm{L}$ ALP for 6 hours at $37^{\circ} \mathrm{C}$ and immunoblotted with Tau 5 after Phos-tag SDS-PAGE. The left lane is the brain extract before ALP treatment (-). Densitometric scans of control and dephosphorylated tau are shown at the right, black for control and gray for dephosphorylated tau. C: Age-dependent isoform expression and phosphorylation of tau in mouse brains. Brain extracts were prepared from mice at ages of P2, 10W, and 96W. Phosphorylation of tau was assessed by immunoblotting with Tau5, RD3, and RD4 after Laemmli (upper panels) or Phos-tag SDS-PAGE (lower panels). The right panel of RD3 is a longer exposure of the second lane (10W long). D: In vitro phosphorylation and dephosphorylation of tau in the brain extracts examined with the use of Phos-tag SDS-PAGE. Brain extracts were incubated at $37^{\circ} \mathrm{C}$ for the indicated times in the absence $(-)$ or presence of ATP and MC and blotted with Tau5 after Phos-tag SDSPAGE (lower panels). Upper panels are Laemmli SDS-PAGE. E: In vitro phosphorylation of recombinant 1 N3R and 1 N4R tau in the P2 and 96W brain extracts were examined with Phos-tag SDS-PAGE. Recombinant tau was incubated with brain extracts at $37^{\circ} \mathrm{C}$ for the indicated times in the presence of ATP and MC and blotted with anti-human tau HT7 after Phos-tag SDS-PAGE. HT7-reactive non-tau band is indicated by arrow in lane of time 0 in the adult brain extract. ALP, alkaline phosphatase; ATP, adenosine triphosphate; Lae, Laemmli SDS-PAGE; MC, microcystin LR; Phos, Phos-tag SDS-PAGE; P2, postnatal day 2; RD3, anti-3R tau; RD4, anti-4R tau; $10 \mathrm{~W}, 10$ weeks of age; $96 \mathrm{~W}, 96$ weeks of age.
Changes in tau phosphorylation are caused by perturbation of the balance between tau kinases and phosphatases. Previous results suggest that the higher kinase activity occurs during the neonatal period. ${ }^{38,42}$ We also tested which of the kinases or phosphatases are the main determinants in phosphorylation of perinatal and adult tau by incubating the brain extracts in the absence or presence of adenosine triphosphate and microcystin LR phosphatase inhibitor. Although a mobility change could be detected in Laemmli SDS-PAGE, the shifts were large enough in Phos-tag SDSPAGE that dephosphorylation (Figure 1D) and phosphorylation (Figure 1D) could be tracked easily. The speed and extent of dephosphorylation were higher in the 10W and $96 \mathrm{~W}$ extracts than in the P2 extract. Moreover, tau shifted upward to a greater extent in the $10 \mathrm{~W}$ and $96 \mathrm{~W}$ brain extracts than in the P2 extract in the presence of adenosine triphosphate and microcystin LR, implying that kinase activity is stronger in adult brains. However, it is also possible that adult 4-repeats (4R) tau is more prone to being phosphorylated than perinatal 3-repeats (3R) tau. To investigate this possibility, we phosphorylated recombinant human $3 \mathrm{R}$ and $4 \mathrm{R}$ tau in the $\mathrm{P} 2$ or $96 \mathrm{~W}$ brain extracts in the presence of adenosine triphosphate and microcystin LR (Figure 1E). To detect human tau specifically, we used antihuman tau antibody HT7. Although HT7 did not react with endogenous mouse tau, there was a HT7 reactive band with higher molecular weight only in the $96 \mathrm{~W}$ extract (Figure 1E), which was also phosphorylated by incubation with adenosine triphosphate and microcystin LR. When the phosphorylation was assessed by the upward shift, interestingly it was found that $3 \mathrm{R}$ and $4 \mathrm{R}$ tau were differently phosphorylated; $4 \mathrm{R}$ tau was phosphorylated faster and more than 3R tau in the P2 and 96W extracts. Furthermore, the $96 \mathrm{~W}$ extract showed similar or slightly higher phosphorylating activity to both $3 \mathrm{R}$ and $4 \mathrm{R}$ tau. Taken together, these results suggest that lower phosphorylation of tau in 


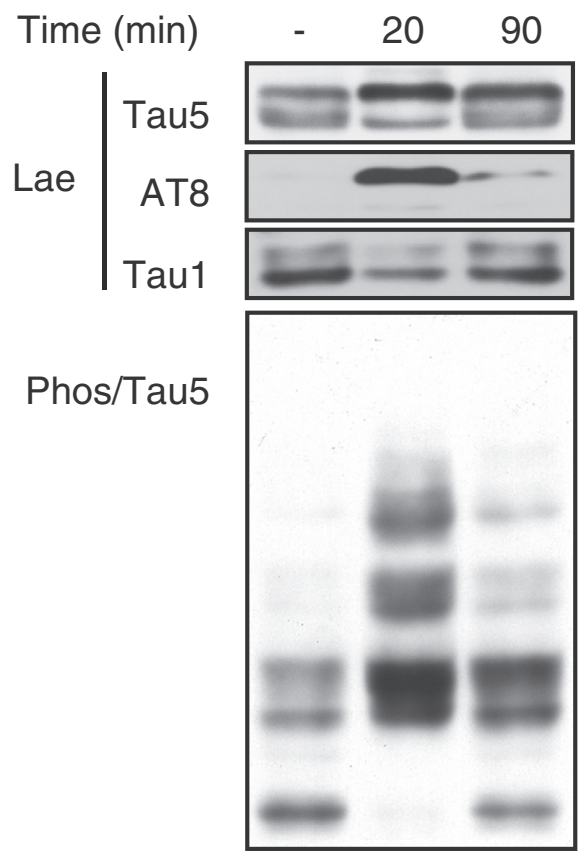

Figure 2 Phosphorylation of tau in cold water-treated mice examined by Phos-tag SDS-PAGE. Mice at 6 weeks of age were treated with ice-cold water for 5 minutes and allowed to recover for 20 or 90 minutes at room temperature. Their brains were dissected, and tau was immunoblotted with Tau5, AT8, and Tau1 after Laemmli SDS-PAGE (upper three panels) or with Tau5 after Phos-tag SDS-PAGE (bottom panel). The control extract from a mouse brain collected without cold-water treatment is shown in the left lane. Lae, Laemmli SDS-PAGE; Phos, Phos-tag SDS-PAGE.

adult brain is because of higher protein phosphatase activity but not kinase activity. ${ }^{43-45}$

\section{Phosphorylation of Tau in Mice Exposed to Cold-Water Stress}

Cold-water stress induces hyperphosphorylation of tau by reducing phosphatase activity. ${ }^{32,33,46}$ With the use of Phos-tag SDS-PAGE, we examined hyperphosphorylation of tau induced in mice by exposure to cold water for a 5 -minute period, followed by varying recovery times of 20 and 90 minutes. The increased phosphorylation was confirmed at Ser199/Ser202/Thr205 by immunoblotting with AT8 (this antibody detects phosphorylated tau) and Tau1 (this antibody detects unphosphorylated tau) (Figure 2). The effect of the cold-water treatment was clearly observed by an upward shift of overall tau species in Phos-tag SDS-PAGE on detection with Tau5 (Figure 2). The lowest nonphosphorylated band disappeared, and upper phosphorylated bands increased at 20 minutes of recovery after the cold-water treatment. After 90 minutes, the tau bands mostly returned to the level observed before the treatment. These results show that cold-water stress increases phosphorylation of all tau species in mouse brain.
Phosphorylation of Tau in Brains of AD Model Mice Analyzed by Phos-Tag SDS-PAGE

We next examined the phosphorylation states of human P301L tau expressed in brains of JNPL3 transgenic mice, in which aggregates of the mutant tau are detected in animals $>6.5$ months of age (approximately $26 \mathrm{~W}$ ) ${ }^{26,47} \mathrm{We}$ assessed mice at $24 \mathrm{~W}$ and $49 \mathrm{~W}$. Sarkosyl-insoluble aggregated tau was found in JNPL3 mice at $49 \mathrm{~W}$ but not at $24 \mathrm{~W}$ (Figure 3A). Although sarkosyl-insoluble tau migrated as a single band slightly slower than sarkosyl-soluble tau in Laemmli SDS-PAGE (Figure 3A), it displayed smeared bands in the upper region of the Phos-tag SDS-PAGE gel (Figure 3A), confirming hyperphosphorylation of sarkosylinsoluble tau. The sarkosyl-insoluble fraction contained a small amount of tau with normal phosphorylation states (Figure 3A), which might represent soluble tau trapped in aggregates. In contrast, soluble tau showed a banding pattern similar to that at $24 \mathrm{~W}$ before aggregate formation (Figure 3A). These results indicate that sarkosyl-soluble P301L tau is not hyperphosphorylated in the brain even when tau aggregates are formed.

We also examined the phosphorylation states of endogenous tau in another mouse AD model, $5 \times \mathrm{FAD}$, which has transgenes of human amyloid precursor protein and presenilin-1 with five familial $\mathrm{AD}$ mutations, leading to increased amyloid $\beta$ peptide production. ${ }^{28}$ Increased amyloid $\beta$ peptide production is reported to induce tau hyperphosphorylation. ${ }^{9,48}$ Although an increase in tau phosphorylation is not detected in this mutant mouse when probed with AT $8,{ }^{28}$ it may be detected if the total tau phosphorylation status is analyzed by Phos-tag SDS-PAGE. Therefore, we examined its phosphorylation at the ages of $12 \mathrm{~W}$

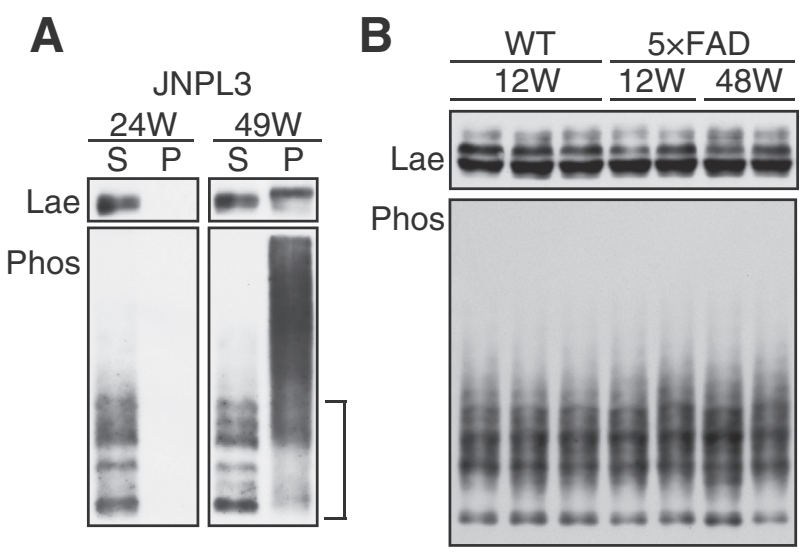

Figure 3 Phosphorylation of tau in brains from two mouse models of Alzheimer disease examined by Phos-tag SDS-PAGE. A: Phosphorylation of human tau in brains of JNPL3 mice at $24 \mathrm{~W}$ and $49 \mathrm{~W}$. Tau was separated into sarkosyl-insoluble and -soluble fractions and detected by immunoblotting with Tau5 after Laemmli (upper panel) or Phos-tag (lower panel) SDS-PAGE. B: Phosphorylation of endogenous tau in brains of $5 \times$ FAD mice at $12 \mathrm{~W}$ and 48W. Tau was detected by immunoblotting with Tau5 after Laemmli (upper panel) or Phos-tag (lower panel) SDS-PAGE. Lae, Laemmli SDS-PAGE; P, sarkosyl-insoluble; Phos, Phos-tag SDS-PAGE; S, sarkosyl-soluble; WT, wildtype mice; 12W, 12 weeks of age; 24W, 24 weeks of age; 48W, 48 weeks of age; 49 W, 49 weeks of age. 

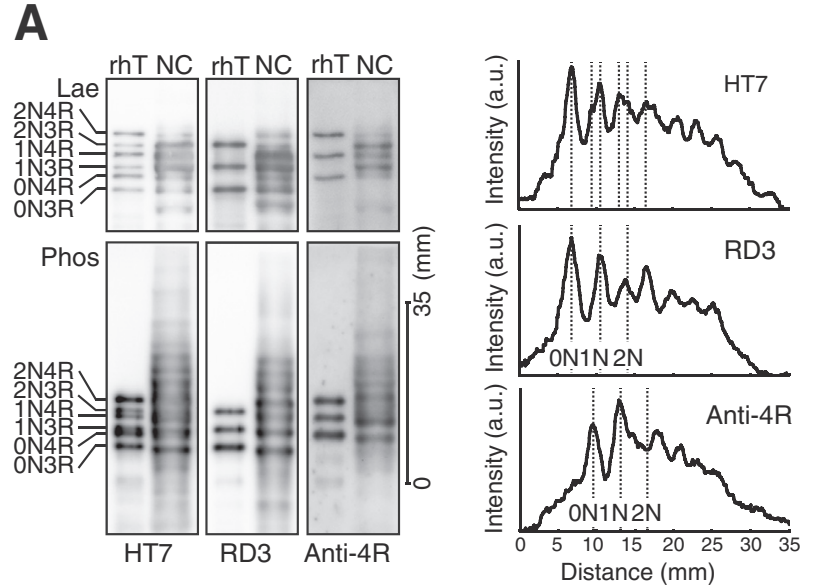

B

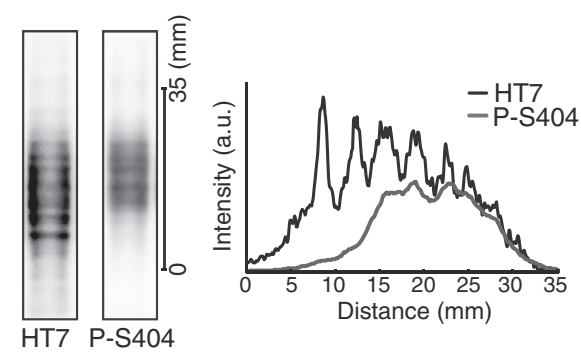

Figure 4 Phosphorylation states of tau in an elderly normal human brain. A: Phosphorylation states of tau in the extract of the brain of an elderly NC (case e) were analyzed by immunoblotting with HT7, RD3, and anti-4R after Laemmli (upper panels) or Phos-tag (lower panels) SDS-PAGE. The six rhTs are shown in the left lane. Densitometric scans of the NC human brain in the Phos-tag SDS-PAGE gels are shown at the right. Dotted vertical lines indicate the positions of the rhTs. The region scanned is indicated at the right side of the blots. B: Ser404 phosphorylation of tau in human brain. Tau in the brain extract of the NC (case e) was immunoblotted with HT7 and P-S404 after Phostag SDS-PAGE; the densitometric scans are shown at the right. Black indicates HT7; gray, P-S404. a.u., arbitrary unit; Lae, Laemmli SDS-PAGE; NC, normal control; Phos, Phos-tag SDS-PAGE; P-S404, phospho-Ser404; RD3, anti-3R tau; $\mathrm{rhT}$, recombinant isoform of human tau.

and 48W, when amyloid plaques and neuron loss, respectively, are reported in the mutant mice. ${ }^{28}$ Tau at both ages migrated almost identically to tau of wild-type mice (Figure 3B), indicating that hyperphosphorylated tau is scarcely present in $5 \times$ FAD mice.

\section{Phosphorylation States of Tau in Human Brains}

Before examination of tau in tauopathy brains, we compared the banding pattern of tau in a normal human brain at 77 years of age (Table 1 ) with the six recombinant human tau isoforms (2N4R, 2N3R, 1N4R, 1N3R, 0N4R, 0N3R). In Laemmli SDS-PAGE, normal human tau showed several bands within the region where recombinant tau migrated (Figure 4A). Human adult brain expresses both $3 R$ and $4 R$ tau. ${ }^{4,6,12,49-51}$ Immunoblotting with $3 \mathrm{R}$ - or $4 \mathrm{R}$-specific antibodies revealed expression of the $0 \mathrm{~N}$ and $1 \mathrm{~N}$ isoforms of both $3 \mathrm{R}$ and $4 \mathrm{R}$ tau, whereas the $2 \mathrm{~N}$ isoforms were less abundant (Figure 4A), consistent with previous results. ${ }^{14,15}$ To avoid weak reactivity of RD4 with human
$4 \mathrm{R}$ tau owing to deamidation, ${ }^{29}$ we used anti-4R tau to detect human $4 \mathrm{R}$ tau. ${ }^{24}$

The six isoforms of recombinant tau were also separated according to their sizes in Phos-tag SDS-PAGE (Figure 4A and Supplemental Figure S1), as in Laemmli SDS-PAGE (Figure 4A), suggesting that nonphosphorylated tau shows the same mobility as the corresponding isoform of recombinant tau. Human tau appeared as at least eight distinct bands with smear staining in the upper region when probed with anti-human tau HT7 (Figure 4A). The Phos-tag blot of normal human brain showed immunoreactivity in the region above recombinant tau, whereas the Laemmli blot did not. Four of the more rapidly migrating bands in Phos-tag SDSPAGE showed mobilities similar to the recombinant $0 \mathrm{~N}$ and $1 \mathrm{~N}$ isoforms of both $3 \mathrm{R}$ and $4 \mathrm{R}$ tau, although brain tau migrated slightly faster than the corresponding recombinant tau bands. To see if some of these bands contain proteolytic fragments of tau isoforms with a larger molecular size, we performed immunoblotting with the Tau-C antibody, whose epitope is in the extreme C-terminal region of tau. ${ }^{23}$ Tau-C showed the similar banding pattern to that of HT7 within the molecular weight range that encompassed the main four tau bands (Supplemental Figure S2). The results suggest that the overlap by truncated tau is, if present, small. Several bands were found in the low molecular weight region, which were recognized by HT7 but not Tau-C. These might be tau fragments without the C-terminal region.

To distinguish $3 R$ and $4 R$ tau, we blotted them with RD3 and anti-4R tau (Figure 4A). 3R tau showed a banding pattern similar to total tau as detected with HT7. These blots suggested that the second and third bands from the bottom might correspond to $0 \mathrm{~N} 4 \mathrm{R} / 1 \mathrm{~N} 3 \mathrm{R}$ and $1 \mathrm{~N} 4 \mathrm{R}$, respectively. To confirm that the upper bands were phosphorylated, immunoblotting with anti-P-S404 was performed (Figure 4B). As expected, the P-S404 immunoreaction was observed only with the bands at the upper region of the blot compared with HT7 immunoblotting. Interestingly, a densitometric profile of the P-S404 reaction almost overlapped with that of HT7 in the upper region; P-S404 accounted for approximately 57\% of total tau (Figure 4B), which agrees well with a mass spectrometry analysis of adult rat brains showing that $56 \%$ of tau was phosphorylated at Ser $404 .^{52}$

\section{Phosphorylation of Tau in Brains of Human Tauopathy Patients}

To gain insight into the pathologic changes in tau, tau in brains of AD and CBD patients was examined by Laemmli and Phos-tag SDS-PAGEs. Immunoblotting with HT7 and AT8 after Laemmli SDS-PAGE is shown in Supplemental Figure S3. AT8 consistently detected the upper tau band [s] in the temporal lobe of AD brains at Braak stage VI. Immunoblotting with HT7 after Phos-tag SDS-PAGE is shown in Figure 5A. All six normal controls showed similar banding patterns (Figure 5A), suggesting that the above results on case e (Figure 4) are representative of the human 

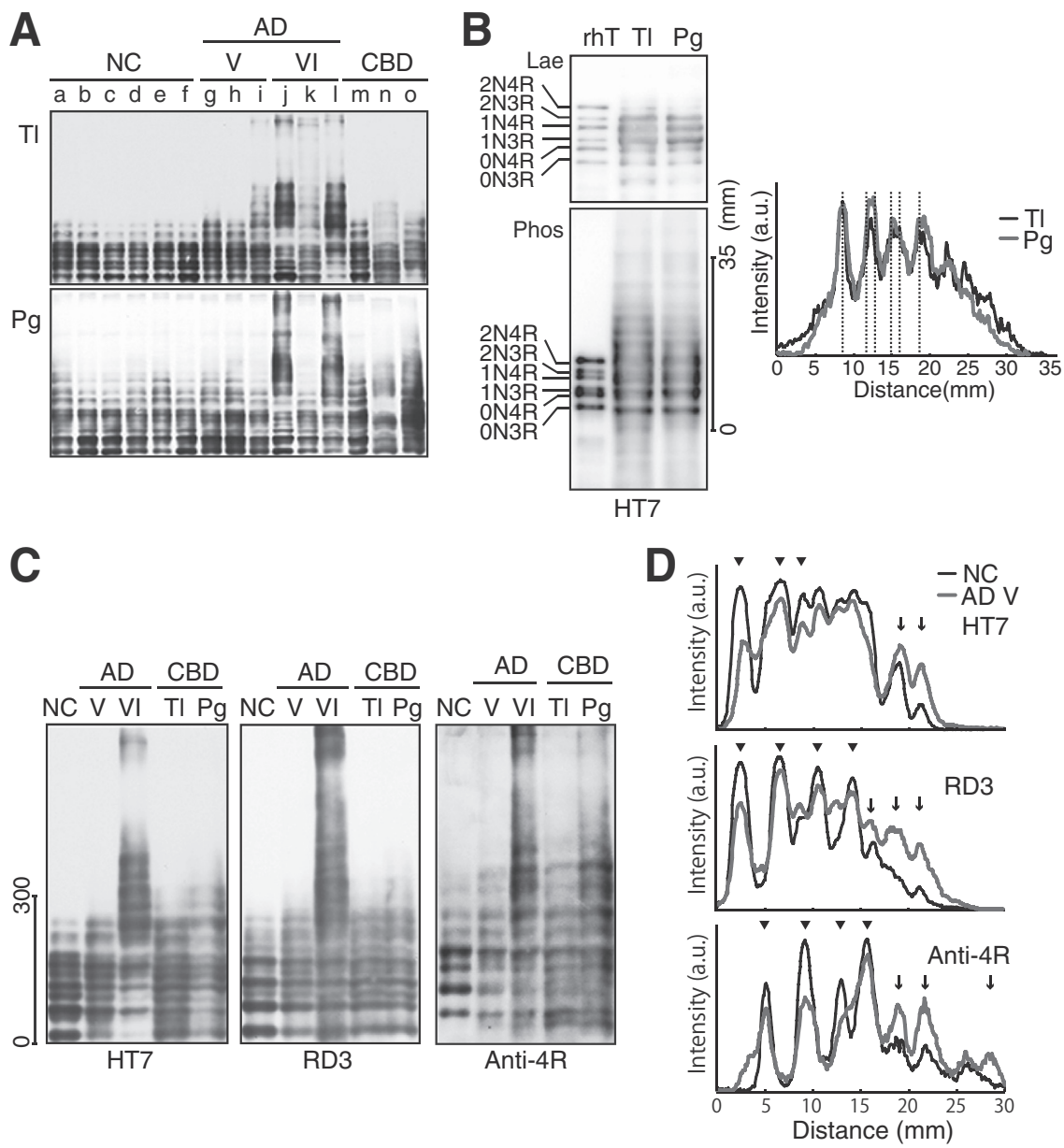

Figure 5 Phosphorylation of tau in brains of human individuals, including NCs and $A D$ and $C B D$ patients. A: Phosphorylation states of tau in human brains. The extracts of the $\mathrm{Tl}$ (upper panel) and $\mathrm{Pg}$ (lower panel) prepared from NCs $(a-f), A D$ $V$ patients $(g-i)$ and AD VI patients $(j-l)$, and CBD patients $(m-0)$ were analyzed by immunoblotting with HT7 after Phos-tag SDS-PAGE. B: Comparison of tau phosphorylation between the temporal lobe and precentral gyrus of a NC individual (case e) after Laemmli (upper panel) and Phos-tag (lower panel) SDS-PAGE. The six rhTs are shown in the left lane. Densitometric scans of the $\mathrm{Tl}$ and $\mathrm{Pg}$ are shown at the right. C: Comparison of tau phosphorylation in human individuals, NC (case e), AD V (case $h$ ) and AD VI (case l), and in the Tl and Pg of CBD (case o) by immunoblotting with HT7, RD3, and anti-4R tau after Phos-tag SDS-PAGE. D: Densitometric scans of tau in NC and AD V blotted with HT7, RD3, and anti-4R. Arrowheads in HT7 indicate nonphospho-tau, which decreases in AD V, and arrowheads in RD3 and anti-4R indicate tau bands that decrease in $A D V$; arrows indicate tau bands increased in $A D$ V. AD, Alzheimer disease; a.u., arbitrary unit; $C B D$, corticobasal degeneration; Lae, Laemmli SDS-PAGE; NC, normal control; $\mathrm{Pg}$, precentral gyrus; Phos, Phos-tag SDS-PAGE; RD3, anti-3R tau; rhT, recombinant isoform of human tau; Tl, temporal lobe; V, Braak stage V; VI, Braak stage VI. brain. In addition to the temporal lobe, which displays primary pathology in $\mathrm{AD}$, the precentral gyrus was examined because that region is affected in CBD. ${ }^{53}$ The banding patterns of tau in the normal controls appeared similar between the temporal lobe and the precentral gyrus, but to compare them more quantitatively, the sample of case e was run on the same blot and subjected to densitometry (Figure 5B). Tau in the temporal lobe was phosphorylated slightly more than that in the precentral gyrus (Figure 5B). $\mathrm{AD}$ patients at Braak stage $\mathrm{V}$ had tau with a little slower migration than the normal control in the temporal lobe (Figure 5A), and tau from patients at Braak stage VI displayed smeared staining in the upper region in both the temporal lobe and precentral gyrus (Figure 5A). CBD patients had a fraction of tau with a slightly slower mobility than tau of normal controls in the temporal lobe, and the amount increased in the precentral gyrus (Figure 5A).

Examples of the normal control (case e) and of $\mathrm{AD}$ at Braak stage V (case h) and VI (case 1), and the temporal lobe and precentral gyrus of CBD (case o) were compared in more detail next to each other with the use of HT7 total tau antibody (Figure 5C). Tau from the AD brain at Braak stage $\mathrm{V}$ and the normal control was analyzed by densitometric scanning (Figure 5D). AD tau at Braak stage $\mathrm{V}$ was shifted upward slightly compared with that in the normal control.
The relative ratio of the nonphosphorylated forms of $0 \mathrm{~N} 3 \mathrm{R}$, 1N3R/0N4R, and 1N4R tau decreased (Figure 5D) and that of upper phosphorylated bands increased in Braak $\mathrm{V}$ AD brain (Figure 5D). This tendency was more remarkable in an AD patient at Braak stage VI, which showed extremely shifted tau (Figure 5C). In a CBD patient, tau had a banding pattern similar to the $\mathrm{AD}$ tau at Braak stage $\mathrm{V}$, and tau species in the precentral gyrus were slightly more phosphorylated than those in the temporal lobe.

Although both $3 R$ and $4 R$ tau isoforms accumulate as neurofibrillary tangles in $\mathrm{AD}, 4 \mathrm{R}$ tau preferentially aggregates in CBD. ${ }^{15,54-56} \mathrm{We}$ asked whether $3 \mathrm{R}$ and $4 \mathrm{R}$ tau isoforms were similarly phosphorylated in $\mathrm{AD}$ or $\mathrm{CBD}$ brains with the use of normal human tau for reference (Figure 5, C and D). The 3R, rather than 4R, tau blot resembled that of total tau (HT7). The signal intensity of AD tau at Braak stage $\mathrm{V}$ increased in the upper bands for both $3 R$ and $4 R$ tau (Figure 5D) and decreased in the lower bands, corresponding to the main bands of the normal control (Figure 5D). Highly phosphorylated smeared tau was detected in both $3 \mathrm{R}$ and $4 \mathrm{R}$ tau at Braak stage VI. Comparison between $3 \mathrm{R}$ and $4 \mathrm{R}$ tau in the CBD patient showed that higher phosphorylated tau was detected in a blot of anti-4R. In addition, 4R tau isoforms were phosphorylated in precentral gyrus more than in the temporal 


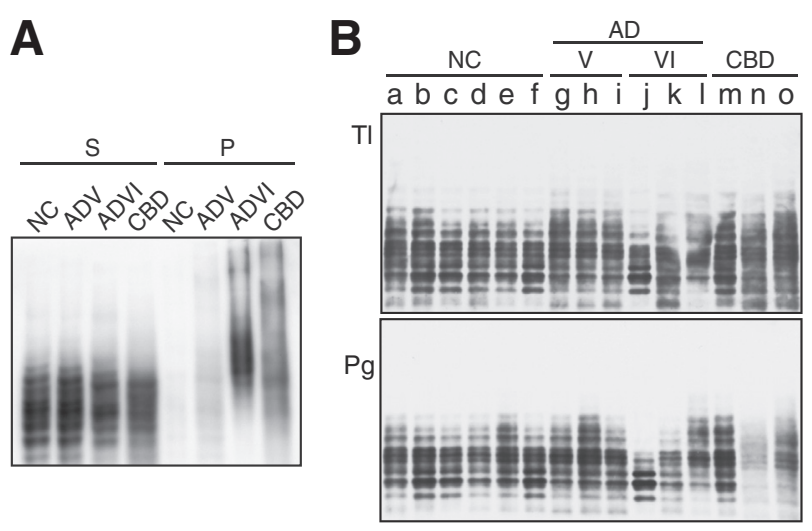

Figure 6 Phosphorylation states of sarkosyl-soluble and -insoluble tau in human tauopathy patients. A: Phos-tag SDS-PAGE immunoblot with HT7 of tau in $S$ and $P$ fractions of human brains. NC, case e; AD V, case h; AD VI, case l; CBD, case 0. B: Phosphorylation states of $S$ tau in the $\mathrm{Tl}$ and $\mathrm{Pg}$ of brains from NC $(a-f), A D V(g-i)$ and $V I(j-l)$, and CBD $(m-0)$ individuals as immunoblotted with HT7 after Phos-tag SDS-PAGE. AD, Alzheimer disease, NC, normal control; $\mathrm{P}$, sarkosyl-insoluble; $\mathrm{Pg}$, precentral gyrus; $\mathrm{S}$, sarkosyl-soluble; Tl, temporal lobe; V, Braak stage V; VI, Braak stage VI.

lobe, whereas phosphorylation states of $3 \mathrm{R}$ tau in $\mathrm{CBD}$ in the temporal lobe and precentral gyrus were similar, consistent with the histologic results (Supplemental Figure S3).

\section{Phosphorylation of Soluble Tau in Brains of Human Tauopathy Patients}

It is not yet known whether hyperphosphorylation of tau begins before or after aggregate formation. ${ }^{2}$ We used the Phos-tag method to detect, if present, the intermediate phosphorylation states of tau in the soluble fraction. Sarkosyl-soluble tau was separated from insoluble tau by centrifugation. ${ }^{57}$ The sarkosyl pellets were suspended in the same volume of SDS sample buffer as the supernatant fluids. Therefore, the immunoblot signals of the pellets represent the amounts of aggregated tau in three individuals. Insoluble tau, which was detected as smeared bands in the upper region of the gel, was increased markedly in $\mathrm{AD}$ at Braak stage VI (Figure 6A). Insoluble tau from a CBD patient was present in an amount intermediate between Braak stage $\mathrm{V}$ and VI of AD patients. The banding pattern of smeared tau appeared different between $\mathrm{AD}$ and CBD.

Soluble tau from normal controls and AD and CBD patients was compared in Figure 6B. Although there were slight differences in the banding patterns, tau from all individuals migrated similarly in Phos-tag SDS-PAGE. The position of the slowest migrating bands, which may represent the most highly phosphorylated species of tau in the soluble fraction, was similar among control and disease brains. Rather, the upper bands of soluble tau in $\mathrm{AD}$ at Braak stage VI (cases j, $\mathrm{k}$, and l) were decreased compared with the control individuals (Figure 6B), suggesting that slightly higher phosphorylated tau in $\mathrm{AD}$ brains at Braak stage V (Figure 5) was already incorporated into aggregates. All of these results suggest that phosphorylation of soluble tau is not increased in $\mathrm{AD}$ or $\mathrm{CBD}$ brains.

Comparison of Highly Phosphorylated Tau in Perinatal Mouse Brains and Brains of Cold Water-Treated Mice with AD Tau

Tau is highly phosphorylated in fetal to perinatal brains (Figure 1C), brains of mice treated with cold-water stress (Figure 2), and $\mathrm{AD}$ brains (Figure 5). However, phosphorylation levels across these three groups were not compared directly. We thus compared the electrophoretic mobilities of tau from these three sources in Phos-tag SDS-PAGE (Figure 7). Interestingly, perinatal tau and cold water-stressed tau showed similar mobilities. A main band in the brain of the cold water-treated mouse migrated to a position similar to one of two main bands in the perinatal brain (Figure 7), suggesting that these tau proteins had similar extents of phosphorylation. In contrast, $\mathrm{AD}$ tau showed a wide range of smeared staining with several recognizable bands in a region similar to visible tau bands in the cold water-stressed brains (Figure 7). These results suggest that tau phosphorylation levels are determined by a similar mechanism in perinatal and cold water-stressed mouse brains, which thus may share some common features with AD brains.

\section{Discussion}

Tau is phosphorylated in normal brains and hyperphosphorylated in AD brains. ${ }^{36,58}$ Although many phosphorylation sites were determined, it is not clear to what extent those sites are phosphorylated under physiologic or pathologic

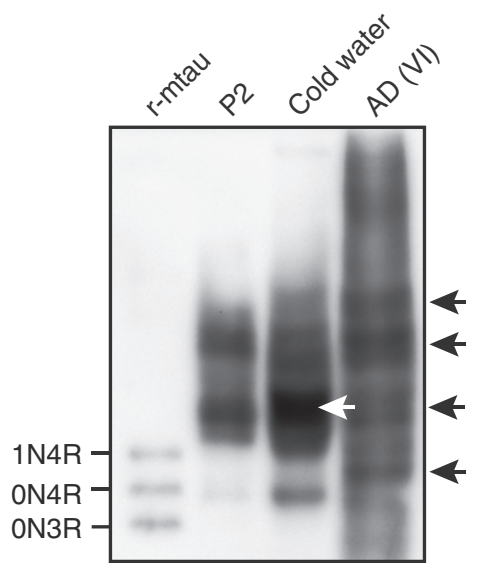

Figure 7 Comparison of phosphorylation states of tau in mouse brain at P2, in the brain from a mouse treated with cold water, and in the brain of an individual with AD VI as detected with Tau 5 antibody after Phos-tag SDS-PAGE. White arrow in the lane of cold water indicates the band with similar migration to a band of neonatal tau. Arrows in the lane of AD VI indicate tau bands migrating within the region of cold water-induced hyperphosphorylated tau. The left lane shows r-mtau 0N3R, 0N4R, and 1N4R. AD, Alzheimer disease; P2, postnatal day 2; r-mtau, recombinant mouse tau isoform; VI, Braak stage VI. 
conditions. In particular, how much tau is nonphosphorylated in brains was not examined. In this study, we applied Phos-tag SDS-PAGE to the analysis of tau phosphorylation in mouse and human brains. The Phos-tag method enabled us to observe the total phosphorylation profiles of tau and revealed that substantial amounts of tau were nonphosphorylated in brains and that sarkosyl-soluble tau was not hyperphosphorylated in the brains from $\mathrm{AD}$ and $\mathrm{CBD}$ patients. These results indicate that tau exists in different phosphorylation states, including totally nonphosphorylated tau, in brains and suggest that hyperphosphorylation proceeds in aggregates.

The upward shift of electrophoretic mobility of tau in Laemmli SDS-PAGE is considered as an indication of phosphorylation, but its use is limited. We have shown here that phosphorylated tau was extremely shifted upward in Phos-tag SDS-PAGE, and the method is useful to observe overall phosphorylation of tau and for quantification of nonphosphorylated tau. It is well known that tau is highly phosphorylated in fetal/perinatal brains ${ }^{35,37,38}$ and in mice exposed to cold-water stress. ${ }^{32,33,46}$ Phos-tag SDS-PAGE not only successfully recapitulated elevated levels of phosphorylation but also systematically demonstrated the entire phosphorylation profile of tau in these contexts. Phos-tag SDS-PAGE also allowed for the first time a comparison of phosphorylation profiles in various contexts in a quantitative manner and suggested a similar mechanism underlying hyperphosphorylation of tau in brains stressed by cold-water treatment and in the perinatal brain (Figure 7). Considering that cold-water treatment preferentially suppresses the activity of protein phosphatases ${ }^{46}$ higher phosphorylation of perinatal tau may also be caused by reduced phosphatase activity, although high kinase activity was suggested. ${ }^{38,42}$ This is supported by the results of in vitro phosphorylation/dephosphorylation experiments (Figure 1, D and E); adult tau, which has a low level of phosphorylation, was phosphorylated in the presence of a phosphatase inhibitor more than perinatal tau, whereas perinatal tau, which has a high endogenous level of phosphorylation, displayed a lower extent of further phosphorylation than adult tau under the same conditions. These data support the idea that the phosphorylation states of tau in mouse brains could be determined primarily by the activity of protein phosphatase(s). ${ }^{45,59}$

Although many sites are phosphorylated in tau in vivo, it is not known whether these sites are phosphorylated in a single tau molecule or in different tau molecules. ${ }^{60}$ Tau was separated into more than four bands in adult mouse brains and more than eight bands in elderly human brains. Considering that proteins are shifted upward depending on the number and sites of phosphorylation in Phos-tag SDSPAGE, ${ }^{21,34}$ these data indicate clearly that there are many tau species with different phosphorylation states. This was also the case when tau was highly phosphorylated in perinatal brains or brains of cold water-stressed mice. AD brains displayed a smear distribution of highly phosphorylated tau in Phos-tag SDS-PAGE. A similar smear reaction was seen in Laemmli SDS-PAGE, but the principle behind the upward shift of tau during electrophoresis is different. Although we could not determine whether these smear bands were a result of phosphorylation and/or aggregates, Phos-tag SDS-PAGE of $\mathrm{AD}$ tau followed by immunoblotting showed several discrete bands, suggesting that a particular combination of sites is phosphorylated in $\mathrm{AD}$ tau. It will be interesting and important to analyze in more detail which sites are phosphorylated when and where in both control and $\mathrm{AD}$ brains.

Phosphorylation, including tyrosine phosphorylation, shifts the proteins upward in Phos-tag SDS-PAGE ${ }^{21,34}$; therefore, a nonphosphorylated component can be separated from the phosphorylated populations. This enabled us to identify nonphosphorylated tau and to measure its ratio in total tau, regardless of the identification of phosphorylation sites. We used bacterially expressed tau for the reference for nonphosphorylated tau. Recombinant human tau isoforms were separated in the order of molecular size in Phos-tag SDS-PAGE (Supplemental Figure S1). Therefore, tau with electrophoretic mobility similar to recombinant tau was considered to be nonphosphorylated. Moreover, because a single antibody was used to detect different phosphorylation states of tau, we can estimate the ratio of tau with different phosphorylation states on a single blot.

One of the interesting findings in this study was the substantial amount of nonphosphorylated tau in mouse and human brains. Detection of totally unphosphorylated tau was lacking in previous studies in which antibodies for phosphorylated tau were mostly used. In contrast, biochemical studies usually estimate the average number of phosphorylation sites, for example, as 1 to 3 phosphates per normal tau and 5 to 10 phosphates per $\mathrm{AD}$ tau. ${ }^{58,61,62} \mathrm{We}$ measured the extent of nonphospho-tau to be approximately $31 \%$ in adult JPNL3 mouse brain expressing the single isoform of human tau. Adult mice endogenously express two isoforms, $0 \mathrm{~N} 4 \mathrm{R}$ and $1 \mathrm{~N} 4 \mathrm{R},{ }^{14}$ of which nonphosphorylated 0N4R tau constituted approximately $30.9 \%$ of total tau. The case of human brain is more complicated because all six isoforms are expressed. ${ }^{15}$ When $3 R$ and $4 R$ tau were measured separately, nonphospho-0N3R tau was $17.9 \%$ of total $3 \mathrm{R}$ tau, and nonphospho-0N4R tau was approximately $14.1 \%$ of total $4 \mathrm{R}$ tau in a normal elderly individual (case e). We cannot exclude the possibility of dephosphorylation in human brains during postmortem delay, but a similar estimate was obtained with mouse brain, (Figure 1) from which tau was prepared in cold buffer that contained phosphatase inhibiters immediately after decapitation. A potential problem is if the proteolytic fragments of tau isoforms with a higher molecular weight overlap nonphosphotau with a smaller molecular size. Tau is targeted by many proteases, including caspase, calpain, and asparagine endopeptidase. ${ }^{63-67}$ Calpain and asparagine endopeptidase produce fragments at around $17 \mathrm{kDa}, 25 \mathrm{kDa}$, and $35 \mathrm{kDa}$ smaller than the smallest 0N3R tau (Supplemental Figure S2). Caspase cleaves tau at Asp421 to generate the C-terminal truncated fragment, ${ }^{63}$ which could have the similar mobility to nonphosphorylated tau. However, this seems unlikely because 
the anti-Tau-C blot gave the similar banding intensity to the HT7 antibody blot. Therefore, we think that substantial amounts of tau are indeed present in a totally nonphosphorylated status in human brain.

We sought to identify the intermediate phosphorylation states of tau, which may represent an early step of hyperphosphorylation. At least to our knowledge, such intermediate phosphorylation states of tau were not described clearly. In addition, it is not yet known whether hyperphosphorylation occurs first on soluble or aggregated tau. ${ }^{2}$ Here, we detected the increase in a slightly higher phosphorylated tau in $\mathrm{AD}$ at Braak stage $\mathrm{V}$ than in the normal control. Such tau was removed from the soluble supernatant fluid of $\mathrm{AD}$ brain extracts by ultracentrifugation, suggesting that the slightly higher phosphorylated tau is already included in aggregates. Although we could not identify these phosphorylated forms in the sarkosyl-insoluble fraction because of larger amounts of aggregated tau, we could conversely compare the phosphorylation profiles of soluble tau in normal, $\mathrm{AD}$, and CBD brains. We found that higher phosphorylation did not occur in the soluble tau in either AD or CBD brains (Figure 6), in agreement with a biochemical study, ${ }^{58}$ in which the same conclusion was derived from detailed elution profiles of proteolytic fragments of soluble tau prepared from normal and AD brains in liquid chromatography. The same results were obtained with FTDP-17 mutant tau in the JNPL3 mouse brain; soluble P301L tau from older mice that contained aggregates in the insoluble fraction showed a phosphorylation pattern identical to tau in younger mice that did not exhibit aggregation (Figure 3A). These results suggest that hyperphosphorylation occurs after incorporation of tau into aggregates. Otherwise, once soluble tau is highly phosphorylated, it may be incorporated into the aggregates immediately in diseased brains. It will be important to identify such intermediate phosphorylation states of tau to understand the initial stage of AD pathogenesis and its development at early stages.

\section{Acknowledgments}

We thank Dr. Yuri Miura (Tokyo Metropolitan Institute for Gerontology) for providing aged C57BL/6J mice, Dr. Masato Taoka (Tokyo Metropolitan University) for discussion, and Dr. Kanae Ando-Iijima for reading the manuscript.

\section{Supplemental Data}

Supplemental material for this article can be found at http://dx.doi.org/10.1016/j.ajpath.2015.10.009.

\section{References}

1. Ballatore C, Lee VM, Trojanowski JQ: Tau-mediated neurodegeneration in Alzheimer's disease and related disorders. Nat Rev Neurosci 2007, 8:663-672
2. Spillantini MG, Goedert M: Tau pathology and neurodegeneration. Lancet Neurol 2013, 12:609-622

3. Lee VM, Goedert M, Trojanowski JQ: Neurodegenerative tauopathies. Annu Rev Neurosci 2001, 24:1121-1159

4. Hutton $\mathrm{M}$, Lendon $\mathrm{CL}$, Rizzu $\mathrm{P}$, Baker $\mathrm{M}$, Froelich $\mathrm{S}$, Houlden H, et al: Association of missense and 5'-splice-site mutations in tau with the inherited dementia FTDP-17. Nature 1998, 393:702-705

5. Poorkaj P, Bird TD, Wijsman E, Nemens E, Garruto RM, Anderson L, Andreadis A, Wiederholt WC, Raskind M, Schellenberg GD: Tau is a candidate gene for chromosome 17 frontotemporal dementia. Ann Neurol 1998, 43:815-825

6. Spillantini MG, Bird TD, Ghetti B: Frontotemporal dementia and Parkinsonism linked to chromosome 17: a new group of tauopathies. Brain Pathol 1998, 8:387-402

7. Bramblett GT, Goedert M, Jakes R, Merrick SE, Trojanowski JQ, Lee VM: Abnormal tau phosphorylation at Ser396 in Alzheimer's disease recapitulates development and contributes to reduced microtubule binding. Neuron 1993, 10:1089-1099

8. Alonso AC, Grundke-Iqbal I, Iqbal K: Alzheimer's disease hyperphosphorylated tau sequesters normal tau into tangles of filaments and disassembles microtubules. Nat Med 1996, 2:783-787

9. Chauhan NB, Siegel GJ, Feinstein DL: Propentofylline attenuates tau hyperphosphorylation in Alzheimer's Swedish mutant model Tg2576. Neuropharmacology 2005, 48:93-104

10. Hanger DP, Anderton BH, Noble W: Tau phosphorylation: the therapeutic challenge for neurodegenerative disease. Trends Mol Med 2009, 15:112-119

11. Mandelkow EM, Mandelkow E: Biochemistry and cell biology of tau protein in neurofibrillary degeneration. Cold Spring Harb Perspect Med 2012, 2:a006247

12. Goedert M, Spillantini MG, Potier MC, Ulrich J, Crowther RA: Cloning and sequencing of the cDNA encoding an isoform of microtubule-associated protein tau containing four tandem repeats: differential expression of tau protein mRNAs in human brain. EMBO J 1989, 8:393-399

13. Janke C, Beck M, Holzer M, Bigl V, Arendt T: Analysis of the molecular heterogeneity of the microtubule-associated protein tau by two-dimensional electrophoresis and RT-PCR. Brain Res Brain Res Protoc 2000, 5:231-242

14. Takuma H, Arawaka S, Mori H: Isoforms changes of tau protein during development in various species. Brain Res Dev Brain Res 2003, 142:121-127

15. Goedert M, Spillantini MG, Cairns NJ, Crowther RA: Tau proteins of Alzheimer paired helical filaments: abnormal phosphorylation of all six brain isoforms. Neuron 1992, 8:159-168

16. Avila J: The tau code. Front Aging Neurosci 2009, 1:1

17. Iqbal K, Grundke-Iqbal I: Developing pharmacological therapies for Alzheimer disease. Cell Mol Life Sci 2007, 64:2234-2244

18. Gustke N, Steiner B, Mandelkow EM, Biernat J, Meyer HE, Goedert M, Mandelkow E: The Alzheimer-like phosphorylation of tau protein reduces microtubule binding and involves Ser-Pro and Thr-Pro motifs. FEBS Lett 1992, 307:199-205

19. Mercken M, Vandermeeren M, Lubke U, Six J, Boons J, Van de Voorde A, Martin JJ, Gheuens J: Monoclonal antibodies with selective specificity for Alzheimer Tau are directed against phosphatase-sensitive epitopes. Acta Neuropathol 1992, 84: $265-272$

20. Tepper K, Biernat J, Kumar S, Wegmann S, Timm T, Hubschmann S, Redecke L, Mandelkow EM, Muller DJ, Mandelkow E: Oligomer formation of tau protein hyperphosphorylated in cells. J Biol Chem 2014, 289:34389-34407

21. Kinoshita E, Kinoshita-Kikuta E, Takiyama K, Koike T: Phosphatebinding tag, a new tool to visualize phosphorylated proteins. Mol Cell Proteomics 2006, 5:749-757

22. Hosokawa T, Saito T, Asada A, Fukunaga K, Hisanaga S: Quantitative measurement of in vivo phosphorylation states of $\mathrm{Cdk} 5$ 
activator p35 by Phos-tag SDS-PAGE. Mol Cell Proteomics 2010, 9 : $1133-1143$

23. Ishiguro K, Sato K, Takamatsu M, Park J, Uchida T, Imahori K: Analysis of phosphorylation of tau with antibodies specific for phosphorylation sites. Neurosci Lett 1995, 202:81-84

24. Hasegawa M, Watanabe S, Kondo H, Akiyama H, Mann DM, Saito Y, Murayama S: 3R and 4R tau isoforms in paired helical filaments in Alzheimer's disease. Acta Neuropathol 2014, 127:303-305

25. Kimura T, Tsutsumi K, Taoka M, Saito T, Masuda-Suzukake M, Ishiguro K, Plattner F, Uchida T, Isobe T, Hasegawa M, Hisanaga S: Isomerase Pin1 stimulates dephosphorylation of tau protein at cyclindependent kinase (Cdk5)-dependent Alzheimer phosphorylation sites. J Biol Chem 2013, 288:7968-7977

26. Lewis J, McGowan E, Rockwood J, Melrose H, Nacharaju P, Van Slegtenhorst M, Gwinn-Hardy K, Paul Murphy M, Baker M, Yu X, Duff K, Hardy J, Corral A, Lin WL, Yen SH, Dickson DW, Davies P, Hutton M: Neurofibrillary tangles, amyotrophy and progressive motor disturbance in mice expressing mutant (P301L) tau protein. Nat Genet 2000, 25:402-405

27. Hosokawa M, Arai T, Masuda-Suzukake M, Nonaka T, Yamashita M, Akiyama H, Hasegawa M: Methylene blue reduced abnormal tau accumulation in P301L tau transgenic mice. PLoS One 2012, 7:e52389

28. Oakley H, Cole SL, Logan S, Maus E, Shao P, Craft J, GuillozetBongaarts A, Ohno M, Disterhoft J, Van Eldik L, Berry R, Vassar R: Intraneuronal beta-amyloid aggregates, neurodegeneration, and neuron loss in transgenic mice with five familial Alzheimer's disease mutations: potential factors in amyloid plaque formation. J Neurosci 2006, 26:10129-10140

29. Dan A, Takahashi M, Masuda-Suzukake M, Kametani F, Nonaka T, Kondo H, Akiyama H, Arai T, Mann DM, Saito Y, Hatsuta H, Murayama S, Hasegawa M: Extensive deamidation at asparagine residue 279 accounts for weak immunoreactivity of tau with RD4 antibody in Alzheimer's disease brain. Acta Neuropathol Commun 2013, 1:54

30. Braak H, Braak E: Neuropathological stageing of Alzheimer-related changes. Acta Neuropathol 1991, 82:239-259

31. Murayama S, Saito Y: Neuropathological diagnostic criteria for Alzheimer's disease. Neuropathology 2004, 24:254-260

32. Planel E, Yasutake K, Fujita SC, Ishiguro K: Inhibition of protein phosphatase $2 \mathrm{~A}$ overrides tau protein kinase I/glycogen synthase kinase 3 beta and cyclin-dependent kinase 5 inhibition and results in tau hyperphosphorylation in the hippocampus of starved mouse. J Biol Chem 2001, 276:34298-34306

33. Okawa Y, Ishiguro K, Fujita SC: Stress-induced hyperphosphorylation of tau in the mouse brain. FEBS Lett 2003, 535:183-189

34. Kinoshita E, Kinoshita-Kikuta E, Koike T: Separation and detection of large phosphoproteins using Phos-tag SDS-PAGE. Nat Protoc 2009, 4:1513-1521

35. Brion JP, Smith C, Couck AM, Gallo JM, Anderton BH: Developmental changes in tau phosphorylation: fetal tau is transiently phosphorylated in a manner similar to paired helical filament-tau characteristic of Alzheimer's disease. J Neurochem 1993, 61: $2071-2080$

36. Matsuo ES, Shin RW, Billingsley ML, Van deVoorde A, O’Connor M, Trojanowski JQ, Lee VM: Biopsy-derived adult human brain tau is phosphorylated at many of the same sites as Alzheimer's disease paired helical filament tau. Neuron 1994, 13:989-1002

37. Morishima-Kawashima M, Hasegawa M, Takio K, Suzuki M, Yoshida H, Titani K, Ihara Y: Proline-directed and non-prolinedirected phosphorylation of PHF-tau. J Biol Chem 1995, 270: 823-829

38. Lovestone $\mathrm{S}$, Reynolds $\mathrm{CH}$ : The phosphorylation of tau: a critical stage in neurodevelopment and neurodegenerative processes. Neuroscience 1997, 78:309-324

39. McMillan P, Korvatska E, Poorkaj P, Evstafjeva Z, Robinson L, Greenup L, Leverenz J, Schellenberg GD, D’Souza I: Tau isoform regulation is region- and cell-specific in mouse brain. J Comp Neurol 2008, 511:788-803

40. de Silva R, Lashley T, Gibb G, Hanger D, Hope A, Reid A, Bandopadhyay R, Utton M, Strand C, Jowett T, Khan N, Anderton B, Wood N, Holton J, Revesz T, Lees A: Pathological inclusion bodies in tauopathies contain distinct complements of tau with three or four microtubule-binding repeat domains as demonstrated by new specific monoclonal antibodies. Neuropathol Appl Neurobiol 2003, 29:288-302

41. de Silva R, Lashley T, Strand C, Shiarli AM, Shi J, Tian J, Bailey KL, Davies P, Bigio EH, Arima K, Iseki E, Murayama S, Kretzschmar H, Neumann M, Lippa C, Halliday G, MacKenzie J, Ravid R, Dickson D, Wszolek Z, Iwatsubo T, Pickering-Brown SM, Holton J, Lees A, Revesz T, Mann DM: An immunohistochemical study of cases of sporadic and inherited frontotemporal lobar degeneration using 3R- and 4R-specific tau monoclonal antibodies. Acta Neuropathol 2006, 111:329-340

42. Takahashi M, Tomizawa K, Ishiguro K: Distribution of tau protein kinase I/glycogen synthase kinase-3beta, phosphatases $2 \mathrm{~A}$ and $2 \mathrm{~B}$, and phosphorylated tau in the developing rat brain. Brain Res 2000, 857:193-206

43. Mawal-Dewan M, Henley J, Van de Voorde A, Trojanowski JQ, Lee VM: The phosphorylation state of tau in the developing rat brain is regulated by phosphoprotein phosphatases. J Biol Chem 1994, 269: 30981-30987

44. Liu F, Grundke-Iqbal I, Iqbal K, Gong CX: Contributions of protein phosphatases PP1, PP2A, PP2B and PP5 to the regulation of tau phosphorylation. Eur J Neurosci 2005, 22:1942-1950

45. Sontag JM, Sontag E: Protein phosphatase 2A dysfunction in Alzheimer's disease. Front Mol Neurosci 2014, 7:16

46. Planel E, Richter KE, Nolan CE, Finley JE, Liu L, Wen Y, Krishnamurthy P, Herman M, Wang L, Schachter JB, Nelson RB, Lau LF, Duff KE: Anesthesia leads to tau hyperphosphorylation through inhibition of phosphatase activity by hypothermia. J Neurosci 2007, 27:3090-3097

47. Sahara N, Lewis J, DeTure M, McGowan E, Dickson DW, Hutton M, Yen SH: Assembly of tau in transgenic animals expressing P301L tau: alteration of phosphorylation and solubility. J Neurochem 2002, $83: 1498-1508$

48. Otth C, Concha II, Arendt T, Stieler J, Schliebs R, GonzalezBillault C, Maccioni RB: AbetaPP induces cdk5-dependent tau hyperphosphorylation in transgenic mice Tg2576. J Alzheimers Dis 2002, 4:417-430

49. Goedert M, Spillantini MG, Jakes R, Rutherford D, Crowther RA: Multiple isoforms of human microtubule-associated protein tau: sequences and localization in neurofibrillary tangles of Alzheimer's disease. Neuron 1989, 3:519-526

50. Kosik KS, Kowall NW, McKee A: Along the way to a neurofibrillary tangle: a look at the structure of tau. Ann Med 1989, 21:109-112

51. Poorkaj P, Kas A, D'Souza I, Zhou Y, Pham Q, Stone M, Olson MV, Schellenberg GD: A genomic sequence analysis of the mouse and human microtubule-associated protein tau. Mamm Genome 2001, 12 : $700-712$

52. Watanabe A, Hasegawa M, Suzuki M, Takio K, MorishimaKawashima M, Titani K, Arai T, Kosik KS, Ihara Y: In vivo phosphorylation sites in fetal and adult rat tau. J Biol Chem 1993, 268: 25712-25717

53. Erbetta A, Mandelli ML, Savoiardo M, Grisoli M, Bizzi A, Soliveri P, Chiapparini L, Prioni S, Bruzzone MG, Girotti F: Diffusion tensor imaging shows different topographic involvement of the thalamus in progressive supranuclear palsy and corticobasal degeneration. AJNR Am J Neuroradiol 2009, 30:1482-1487

54. Ksiezak-Reding H, Morgan K, Mattiace LA, Davies P, Liu WK, Yen SH, Weidenheim K, Dickson DW: Ultrastructure and biochemical composition of paired helical filaments in corticobasal degeneration. Am J Pathol 1994, 145:1496-1508

55. Sergeant N, Wattez A, Delacourte A: Neurofibrillary degeneration in progressive supranuclear palsy and corticobasal degeneration: tau 
pathologies with exclusively "exon 10" isoforms. J Neurochem $1999,72: 1243-1249$

56. Arai T, Ikeda K, Akiyama H, Shikamoto Y, Tsuchiya K, Yagishita S, Beach T, Rogers J, Schwab C, McGeer PL: Distinct isoforms of tau aggregated in neurons and glial cells in brains of patients with Pick's disease, corticobasal degeneration and progressive supranuclear palsy. Acta Neuropathol 2001, 101:167-173

57. Masuda-Suzukake M, Nonaka T, Hosokawa M, Kubo M, Shimozawa A, Akiyama H, Hasegawa M: Pathological alphasynuclein propagates through neural networks. Acta Neuropathol Commun 2014, 2:88

58. Hasegawa M, Morishima-Kawashima M, Takio K, Suzuki M, Titani K, Ihara Y: Protein sequence and mass spectrometric analyses of tau in the Alzheimer's disease brain. J Biol Chem 1992, 267:17047-17054

59. Gong CX, Iqbal K: Hyperphosphorylation of microtubule-associated protein tau: a promising therapeutic target for Alzheimer disease. Curr Med Chem 2008, 15:2321-2328

60. Hernandez F, Lucas JJ, Cuadros R, Avila J: GSK-3 dependent phosphoepitopes recognized by PHF-1 and AT- 8 antibodies are present in different tau isoforms. Neurobiol Aging 2003, 24:1087-1094

61. Ksiezak-Reding H, Liu WK, Yen SH: Phosphate analysis and dephosphorylation of modified tau associated with paired helical filaments. Brain Res 1992, 597:209-219

62. Kopke E, Tung YC, Shaikh S, Alonso AC, Iqbal K, Grundke-Iqbal I: Microtubule-associated protein tau. Abnormal phosphorylation of a non-paired helical filament pool in Alzheimer disease. J Biol Chem 1993, 268:24374-24384

63. Gamblin TC, Chen F, Zambrano A, Abraha A, Lagalwar S, Guillozet AL, Lu M, Fu Y, Garcia-Sierra F, LaPointe N, Miller R, Berry RW, Binder LI, Cryns VL: Caspase cleavage of tau: linking amyloid and neurofibrillary tangles in Alzheimer's disease. Proc Natl Acad Sci U S A 2003, 100:10032-10037

64. Rissman RA, Poon WW, Blurton-Jones M, Oddo S, Torp R, Vitek MP, LaFerla FM, Rohn TT, Cotman CW: Caspase-cleavage of tau is an early event in Alzheimer disease tangle pathology. J Clin Invest 2004, 114:121-130

65. Park SY, Ferreira A: The generation of a $17 \mathrm{kDa}$ neurotoxic fragment: an alternative mechanism by which tau mediates betaamyloid-induced neurodegeneration. J Neurosci 2005, 25: $5365-5375$

66. Basurto-Islas G, Luna-Muñoz J, Guillozet-Bongaarts AL, Binder LI, Mena R, García-Sierra F: Accumulation of aspartic acid421- and glutamic acid391-cleaved tau in neurofibrillary tangles correlates with progression in Alzheimer disease. J Neuropathol Exp Neurol 2008, $67: 470-483$

67. Zhang Z, Song M, Liu X, Kang SS, Kwon IS, Duong DM, Seyfried NT, Hu WT, Liu Z, Wang JZ, Cheng L, Sun YE, Yu SP, Levey AI, Ye K: Cleavage of tau by asparagine endopeptidase mediates the neurofibrillary pathology in Alzheimer's disease. Nat Med 2014, 20:1254-1262 